\title{
Interval Ranges of Fuzzy Sets Induced by Arithmetic Operations Using Gradual Numbers
}

\author{
Qingsong Mao ${ }^{1}$ and Huan Huang ${ }^{2, *}$ \\ 1 Teachers College, Jimei University, Xiamen 361021, China; pinem@163.com or 200261000022@jmu.edu.cn \\ 2 Department of Mathematics, Jimei University, Xiamen 361021, China \\ * Correspondence: hhuangjy@126.com or 200261000004@jmu.edu.cn
}

Citation: Mao, Q.; Huang, $\mathrm{H}$.

Interval Ranges of Fuzzy Sets Induced by Arithmetic Operations Using Gradual Numbers. Mathematics 2021, 9, 1351. https://doi.org/ $10.3390 /$ math 9121351

Academic Editors: Sorin Nadaban and Ioan Dzitac

Received: 13 May 2021

Accepted: 9 June 2021

Published: 11 June 2021

Publisher's Note: MDPI stays neutral with regard to jurisdictional claims in published maps and institutional affiliations.

Copyright: (c) 2021 by the authors. Licensee MDPI, Basel, Switzerland. This article is an open access article distributed under the terms and conditions of the Creative Commons Attribution (CC BY) license (https:/ / creativecommons.org/licenses/by/ $4.0 /)$.

\begin{abstract}
Wu}$ introduced the interval range of fuzzy sets. Based on this, he defined a kind of arithmetic of fuzzy sets using a gradual number and gradual sets. From the point of view of soft computing, this definition provides a new way of handling the arithmetic operations of fuzzy sets. The interval range is an important characterization of a fuzzy set. The interval range is also useful for analyses and applications of arithmetic. In this paper, we present general conclusions on crucial problems related to interval ranges of fuzzy sets induced by this arithmetic. These conclusions indicate that the corresponding conclusions in previous works should be modified: firstly, we give properties of the arithmetic and the composites of finite arithmetic. Then, we discuss the relationship between the domain of a gradual set and the range of its induced fuzzy set, and the relationship between the domain of a gradual set and the interval range of its induced fuzzy set. Based on the above results, we present the relationship between the intersection of the interval ranges of a group of fuzzy sets and the interval ranges of their resulting fuzzy sets obtained by compositions of finite arithmetic. Furthermore, we construct examples to show that even under conditions stronger than in previous work, there are still various possibilities in the relationship between the intersection of interval ranges of a group of fuzzy sets and the ranges of their resulted fuzzy sets, and there are still various possibilities in the relationship between the intersection of the interval ranges of a group of fuzzy sets and the interval ranges of their resulting fuzzy sets.
\end{abstract}

Keywords: interval range; arithmetic; gradual numbers; gradual sets

\section{Introduction}

Since Zadeh [1] put forward the concept of a fuzzy set in 1965, the fuzzy set theory has been widely used to handle uncertainties [2-4]. In 2008, new concepts of "gradual set" and "gradual number" were introduced in fuzzy set theory $[5,6]$. The gradual set and the gradual number are effective tools to study fuzzy sets, and have considerable applications in soft computing and related areas [7-10].

Fortin, Dubois and Fargier [5] introduced the concept of a gradual number and proposed the idea of representing a 1-dimensional fuzzy number as a crisp interval of gradual numbers. Dubois and Prade [6] introduced the concept of a gradual set and put forward the idea of inducing a fuzzy set by a gradual set or even an assignment function.

Fuzzy arithmetic are fundamental and essential in fuzzy set theory and widely used in soft computing. Fuzzy arithmetic and studies related to fuzzy arithmetic have attracted much attention [8,11-16]. For the need of theory and practical applications, various kinds of fuzzy arithmetic are proposed and studied. It is well known that Zadeh's extension principle [17] can be used to induce the arithmetic operations of fuzzy sets.

Interval arithmetic play important roles in many kinds of fuzzy arithmetic. Recently, $\mathrm{Wu}$ [15] proposed a new way to handle the arithmetic operations of fuzzy sets which does not directly use the interval arithmetic. In this paper, we discuss the relationship between the arithmetic defined by $\mathrm{Wu}$ and the interval arithmetic (see Section 3). 
$\mathrm{Wu}$ [15] introduced the concept of the interval range of a fuzzy set. Based on the use of an interval range, he introduced the arithmetic operations of fuzzy sets in $\mathbb{R}$ by using gradual sets and gradual numbers.

According to Wu's definition, the resulted fuzzy sets of arithmetic operations of two fuzzy sets are defined as the induced fuzzy sets of the gradual sets on the intersection of the interval ranges of these two fuzzy sets. These gradual sets were generated from the arithmetic of gradual numbers. Here, we mention that in extreme cases, the gradual sets will be replaced by an assignment function with its value at each element being the empty set. Wu's definition provides a new way of handling the arithmetic operations of fuzzy sets.

The interval range is an important characterization of a fuzzy set. The interval range is also useful for the analyses and applications of this arithmetic. Therefore, the discussions on the interval ranges are essential and important in the study of arithmetic operations given by $\mathrm{Wu}[15]$.

Wu [15] claimed that, under certain conditions, the domain of a gradual set is equal to the range of its induced fuzzy set.

On the basis of this, Wu [15] claimed that the intersection of the interval ranges of two fuzzy sets is equal to the interval ranges of the resulted fuzzy sets obtained by the arithmetic operations of these two fuzzy sets.

Furthermore, $\mathrm{Wu}$ [15] claimed that, under certain conditions, the intersection of the interval ranges of a group of fuzzy sets, the interval ranges and the ranges of their resulted fuzzy sets obtained by compositions of finite arithmetic operations are equal.

In this paper, we pointed out that the above conclusions in [15] should be modified. Based on investigations into the properties of the arithmetic and the composites of finite arithmetic, we further give general conclusions on the above relationships in [15]. Examples are constructed to illustrate various possibilities in the relationships.

Here, we should mention that our results are complementary to [15], not in competition with [15].

The remainder of this paper is organized as follows. Section 2 recalls some basic concepts about fuzzy sets, gradual sets and gradual numbers. Section 3 reviews the arithmetic of fuzzy sets in $\mathbb{R}$ introduced by Wu [15]. Furthermore, we give some properties of the arithmetic and the composites of finite arithmetic. In Sections 4-6, we point out that some conclusions on interval ranges and ranges in [15] should be modified, and have presented general conclusions. Section 4 discusses the domain of a gradual set and the interval range and range of its induced fuzzy set. Section 5 considers the intersection of the interval ranges of a group of fuzzy sets and the interval ranges of their resulted fuzzy sets obtained by compositions of finite arithmetic. Section 6 investigates the intersection of the interval ranges of a group of fuzzy sets and the ranges and interval ranges of their resulted fuzzy sets under certain conditions. Finally, we draw our conclusions in Section 7.

\section{Fuzzy Sets, Gradual Sets and Gradual Numbers}

In this section, we recall some basic concepts about fuzzy sets, gradual sets and gradual numbers. For details, we refer the readers to $[2,5,6,18]$.

Let $X$ be a topology space; that is, $X$ is a non-empty set equipped with a topological structure. A fuzzy set $A$ in $X$ can be identified with its membership function $A: X \rightarrow[0,1]$. We use $F(X)$ to denote the set of all fuzzy sets in $X$.

For a subset $S$ of $X$, we use $\bar{S}_{X}$ to denote the characteristic function of $S$ on $X$, meaning that:

$$
\bar{S}_{X}(x)= \begin{cases}1, & \text { if } x \in S, \\ 0, & \text { if } x \in X \backslash S .\end{cases}
$$

$\bar{S}_{X}$ can be regarded as a fuzzy set in $X$. 
Let $A$ be a fuzzy set in $X$. Then, the $\alpha$-cut set of $A$ is defined as

$$
[A]_{\alpha}= \begin{cases}\{x: A(x) \leq \alpha\}, & \alpha>0 \\ \operatorname{cl}_{X}\{x: A(x)>0\}, & \alpha=0\end{cases}
$$

where $\mathbf{c l}_{X}\{S\}$ denotes the closure of $S$ in $X$.

Let $\mathbb{R}$ be the real number space. Let $S$ be a set of real numbers. In this paper, we use $\operatorname{cl}\{S\}$ to denote $\mathbf{c l}_{\mathbb{R}}\{S\}$ and use $\bar{S}$ to denote the fuzzy set $\bar{S}_{\mathbb{R}}$ in $\mathbb{R}$.

Fuzzy set $A$ is said to be normalif $[A]_{1} \neq \varnothing$. The fuzzy number is a type of normal fuzzy set which has been exhaustively studied $[5,19,20]$. It should be mentioned that, in theoretical research and practical applications, discussions about fuzzy sets are often in frameworks containing non-normal fuzzy sets $[10,15,21]$. In this paper, the discussion is on general fuzzy sets including normal fuzzy sets and non-normal fuzzy sets.

Let $A$ be a fuzzy set in $X$. The range of $A$ is denoted by $\mathcal{R}(A)$. Wu [15] introduced the interval range $I_{A}$ of $A$, which is defined by

$$
I_{A}:= \begin{cases}{[0, \sup \mathcal{R}(A)],} & \text { if } \sup \mathcal{R}(A) \text { is attained; } \\ {[0, \sup \mathcal{R}(A)),} & \text { if } \sup \mathcal{R}(A) \text { is not attained. }\end{cases}
$$

That is to say $I_{A}=\{\alpha \in[0,1]: \alpha \leq \beta$ for some $\beta \in \mathcal{R}(A)\}$.

Dubois and Prade [6] first introduced the concept of gradual set and proposed the idea of using a gradual set to induce a fuzzy set (see Definitions 2 and 4 in [6]).

The definition of a gradual set according to $\mathrm{Wu}$ [15] is different from the definition of a gradual set according to Dubois and Prade [6]. Wu [15] defined the gradual set as follows (see Definition 3.1 in [15]):

- A gradual set $\mu$ of $X$ is an assignment function $\mu: I \rightarrow P(X) \backslash\{\varnothing\}$, where $I$ is a subset of $[0,1]$.

A gradual set of $X$ is a special type of assignment function on $I \subseteq[0,1]$.

We introduce the fuzzy set $F_{\mu}$ induced by an assignment function $\mu: I \rightarrow P(X)$, $I \subseteq[0,1]$, which is defined by

$$
F_{\mu}(x)=\sup \{\alpha \in(0,1] \cap I: x \in \mu(\alpha)\} .
$$

In this paper, we set sup $\varnothing=\mathbf{0}$ by default.

It can be seen that $F_{\mu}$ can also be written as

$$
F_{\mu}(x)=\sup _{\alpha \in I} \alpha \cdot \overline{\mu(\alpha)}_{X}(x)
$$

$\mathrm{Wu}$ [15] defined the fuzzy set induced from a gradual set by (2) (see Equation (10) in [15]).

Remark 1. The way to induce a fuzzy set from an assignment function given by (1) is consistent with the ways to induce a fuzzy set from a gradual set given by Wu [15] and Dubois and Prade [6], respectively. These ways of inducing fuzzy sets essentially come from [6].

Fortin, Dubois and Fargier [5] first introduced the concept of gradual number. They proposed how to represent a 1-dimensional fuzzy number as a crisp interval of gradual numbers.

$\mathrm{Wu}$ [15] slightly modified the concept of gradual number as follows:

- A gradual number $a$ in $\mathbb{R}$ is an assignment function $a: I \rightarrow \mathbb{R}$, where $I$ is a subset of $[0,1]$.

$\mathrm{Wu}$ 's modified definition of a gradual number is more convenient to deal with nonnormal fuzzy sets. 
A gradual number $a$ is said to be an element of a fuzzy set $A$ in $F(\mathbb{R})$, denoted by $a \in A$, if $a$ is defined on $I_{A}$ and $a(\alpha) \in[A]_{\alpha}$ for each $\alpha \in I_{A}$.

\section{Arithmetic of Fuzzy Sets in $F(\mathbb{R})$ Using Gradual Number}

In this section, we review the arithmetic of fuzzy sets in $F(\mathbb{R})$ introduced by Wu [15]. Furthermore, we give properties of the arithmetic and the composites of finite arithmetic, which are useful in this paper.

$\mathrm{Wu}$ [15] introduced the arithmetic operations of two gradual numbers which are elements of two fuzzy sets in $F(\mathbb{R})$, respectively.

Based on this, Wu [15] defined the arithmetic operations of two fuzzy sets in $F(\mathbb{R})$ as follows.

First, a gradual set or an assignment function, which is not a gradual set, is generated from the corresponding arithmetic of gradual numbers in these two fuzzy sets, respectively. The domain of this gradual set or this assignment function is the intersection of interval ranges of these two fuzzy sets. Then, the resulted fuzzy set of the arithmetic operation is induced from this gradual set or this assignment function.

In this paper, we still use,$+-\times, /$ to denote the arithmetic operations of fuzzy sets in $F(\mathbb{R})$ introduced by $\mathrm{Wu}[15]$.

Suppose that $A$ and $B$ are two fuzzy sets in $\mathbb{R}$, and that $\circ \in\{+,-, \times, /\}$ is an arithmetic operation. For two gradual numbers $a \in A$ and $b \in B$, we use $I^{\cap}$ to denote $I_{A} \cap I_{B}$, and the arithmetic operation $a \circ b$ is defined by

$$
(a \circ b)(\alpha)=a(\alpha) \circ b(\alpha)
$$

for all $\alpha \in I^{\cap}$.

$a \circ b$ is said to be meaningful if $\left\{\alpha \in I^{\cap}: a(\alpha) \circ b(\alpha)\right.$ is meaningful $\}=I^{\cap}$, at this time, and $a \circ b$ is a gradual number on $I^{\bigcap}$, otherwise $a \circ b$ is meaningless.

Therefore, taking $\circ \in\{+,-, \times\}$, then $a \circ b$ is meaningful; taking $\circ=/$, then $a \circ b$ is meaningful if and only if the set $M_{b}:=\left\{\alpha \in I^{\cap}: b(\alpha) \neq 0\right\}$ satisfies that $M_{b}=I^{\cap}$.

The family $\{a \circ b: a \in A, b \in B$ with $a \circ b$ is meaningful $\}$ induces an assignment function $\sigma: I^{\cap} \rightarrow P(\mathbb{R})$ given by

$$
\sigma(\alpha)=\{(a \circ b)(\alpha): a \in A, b \in B \text { with } a \circ b \text { is meaningful }\} .
$$

Using this assignment function $\sigma, A \circ B$ is defined by

$$
(A \circ B)(x)=\sup _{\alpha \in I^{\cap}} \alpha \cdot \overline{\sigma(\alpha)}(x) .
$$

From the above definition, $A / B$ is given by

$$
\begin{aligned}
(A / B)(x) & =\sup _{\alpha \in I^{\cap}} \alpha \cdot \overline{\sigma(\alpha)}(x) \\
& =\sup _{\alpha \in I^{\curvearrowleft}} \alpha \cdot \overline{\left\{a(\alpha) / b(\alpha): a \in A, b \in B \text { with } b(\alpha) \neq 0 \text { for all } \alpha \in I^{\cap}\right\}}(x) .
\end{aligned}
$$

Remark 2. Here, we slightly adjust the presentation of the definition of $A \circ B$ to improve the accuracy and clarity of the presentation. Readers may compare it with the corresponding contents in Section 4 of [15].

Remark 3. From (4), we know that $A \circ B=F_{\sigma}$, where $\sigma$ is given by (3).

If the family $\{a \circ b: a \in A, b \in B$ with $a \circ b$ is meaningful $\} \neq \varnothing$, then $\sigma$ is a gradual set from $I^{\cap}$ to $P(\mathbb{R}) \backslash \varnothing$; if the family $\{a \circ b: a \in A, b \in B$ with $a \circ b$ is meaningful $\}=\varnothing$, then $\sigma(\alpha)=\varnothing$ for all $\alpha \in I^{\cap}$.

Let $[A]_{\alpha} \circ[B]_{\alpha}:=\left\{z: z=x \circ y\right.$ with $\left.x \in[A]_{\alpha}, y \in[B]_{\alpha}\right\}$. Then:

(i) If $\circ \in\{+,-, \times\}$, then $\sigma(\alpha)=[A]_{\alpha} \circ[B]_{\alpha}$ for all $\alpha \in I^{\cap}$. 
(ii) If $\circ=/$ and there exists $a b \in B$ satisfying that $b(\alpha) \neq 0$ for all $\alpha \in I^{\cap}$, then $\sigma(\alpha)=[A]_{\alpha} /[B]_{\alpha}$ for all $\alpha \in I^{\cap}$.

(iii) If $\circ=/$ and there is no $b \in B$ satisfying that $b(\alpha) \neq 0$ for all $\alpha \in I^{\cap}$, then $\sigma(\alpha)=\varnothing$ for all $\alpha \in I^{\cap}$.

Proposition 1. Let $A$ and $B$ be fuzzy sets in $F(\mathbb{R})$. Then, $A \circ B=\bar{\varnothing}$ if and only if $A, B$ satisfy one of the following conditions.

(i) $A=\bar{\varnothing}$ or $B=\bar{\varnothing}$; that is $I^{\cap}=\{0\}$.

(ii) $A \neq \bar{\varnothing}, B \neq \bar{\varnothing}, \circ=1$, and there is no $b \in B$ satisfying that $b(\alpha) \neq 0$ for all $\alpha \in I^{\cap}$.

Proof. The desired result follows from the definition of $A \circ B$.

Suppose that $f$ is a composite of the finite arithmetic operations of real numbers. The symbol $\widetilde{f}$ denotes the corresponding composite of finite arithmetic operations of fuzzy sets in $\mathbb{R}$.

For instance, if $f(x, y, z)=(x-y) \times z$ for $x, y, z \in \mathbb{R}$, then $\widetilde{f}(u, v, w)=(u-v) \times w$ for $u, v, w \in F(\mathbb{R})$.

For $\widetilde{f}\left(A_{1}, \ldots, A_{n}\right)$, we use $\boldsymbol{I}^{\cap}$ to denote $\bigcap_{i=\mathbf{1}}^{n} \boldsymbol{I}_{A_{i}}$. If $\widetilde{f}\left(A_{1}, \ldots, A_{n}\right)$ is $A \circ B$, then $I^{\cap}=I_{A} \cap I_{B}$.

The symbol $f\left(\left[A_{1}\right]_{\alpha}, \ldots,\left[A_{n}\right]_{\alpha}\right)$ is used to denote the set:

$$
\left\{z: z=f\left(x_{1}, \ldots, x_{n}\right) \text { with } x_{i} \in\left[A_{i}\right]_{\alpha}, i=1, \ldots, n\right\} .
$$

Theorem 1. Let $f$ be a composite of finite arithmetic operations of real numbers and for each $i=1, \ldots, n$, let $A_{i}$ be a fuzzy set in $F(\mathbb{R})$. Furthermore, let $\mu$ be an assignment function from $I^{\cap}$ to $P(\mathbb{R})$ defined as $\mu(\alpha)=f\left(\left[A_{1}\right]_{\alpha}, \ldots,\left[A_{n}\right]_{\alpha}\right)$.

(i) If $A_{i}=\bar{\varnothing}$ for some $i \in\{1, \ldots, n\}$, i.e, $I^{\cap}=\{0\}$, then $\widetilde{f}\left(A_{1}, \ldots, A_{n}\right)=F_{\mu}=\bar{\varnothing}$.

(ii) If $\widetilde{f}\left(A_{1}, \ldots, A_{n}\right) \neq \bar{\varnothing}$, then $\tilde{f}\left(A_{1}, \ldots, A_{n}\right)=F_{\mu}$.

(iii) If $f$ is a composition of $\circ \in\{+,-, \times\}$, then $\widetilde{f}\left(A_{1}, \ldots, A_{n}\right)=F_{\mu}$.

Proof. From Proposition 1, (i) is true.

If $\widetilde{f}\left(A_{1}, \ldots, A_{n}\right) \neq \bar{\varnothing}$, then $I^{\cap} \supsetneqq\{0\}$. By using Remark 3, we can deduce that $\widetilde{f}\left(A_{1}, \ldots, A_{n}\right)=F_{\mu}$. So (ii) is true.

Suppose that $f$ is a composition of $\circ \in\{+,-, \times\}$. Then $\widetilde{f}\left(A_{1}, \ldots, A_{n}\right)=\bar{\varnothing}$ if and only if $A_{i}=\bar{\varnothing}$ for some $i \in\{1, \ldots, n\}$. Thus, (iii) follows from (i) and (ii).

\section{Ranges and Interval Ranges of Induced Fuzzy Sets}

It is claimed in [15] that under certain kinds of conditions, the domain $I$ of a gradual set $\mu$ is equal to the range $\mathcal{R}\left(F_{\mu}\right)$ of its induced fuzzy set $F_{\mu}$.

In this section, we point out by examples that these conclusions should be modified. $\mathcal{R}\left(F_{\mu}\right) \neq I$ can be divided into three cases: " $\mathcal{R}\left(F_{\mu}\right) \supsetneqq I$, " $\mathcal{R}\left(F_{\mu}\right) \varsubsetneqq I$ ", and " $\mathcal{R}\left(F_{\mu}\right) \varsubsetneqq I$ and $I \nsupseteq \mathcal{R}\left(F_{\mu}\right)^{\prime \prime}$. In fact, we show that even under stronger conditions, all these three cases could happen.

Furthermore, we give the relationship between $I$ and $\mathcal{R}\left(F_{\mu}\right)$, and the relationship between $I$ and the interval range $I_{F_{\mu}}$ of the induced fuzzy set $F_{\mu}$.

The conclusions in this section show that for a gradual set $\mu$ and its induced fuzzy set $F_{\mu}$, the interval range $I_{F_{\mu}}$ and the range $\mathcal{R}\left(F_{\mu}\right)$ of $F_{\mu}$ vary in the scope determined by the domain $I$ of $\mu$, respectively. On the other hand, even under stronger conditions than in [15], both $\mathcal{R}\left(F_{\mu}\right) \neq I$ and $I_{F_{\mu}} \neq I$ could happen.

An assignment function $\mu: I \rightarrow P(X)$ is said to be strictly nested if $\mu(\alpha) \varsubsetneqq \mu(\beta)$ for $\alpha, \beta \in I$ with $\alpha>\beta$.

Affirmation 1 ([15]). (See Remark 3.5 and Proposition 3.6 of [1]) Let $\mu: I \rightarrow P(X) \backslash\{\varnothing\}$ be a gradual set: 
(i) If $I=[0, \alpha]$ or $I=[0, \alpha), \alpha>0$, then $\mathcal{R}\left(F_{\mu}\right)=I$.

(ii) If $\mu$ is strictly nested, then $\mathcal{R}\left(F_{\mu}\right)=I$.

Remark 4. Affirmation 1 is not valid. In fact, even if the gradual set $\mu: I \rightarrow P(\mathbb{R}) \backslash\{\varnothing\}$ satisfies:

(i') $I=[0, \alpha]$ or $I=[0, \alpha), \alpha>0$, and

(ii') $\mu$ is strictly nested,

all the three cases " $\mathcal{R}\left(F_{\mu}\right) \supsetneqq I$ ", “ $\mathcal{R}\left(F_{\mu}\right) \varsubsetneqq I^{\prime}$, and “ $\mathcal{R}\left(F_{\mu}\right) \nsupseteq I$ and $I \nsupseteq \mathcal{R}\left(F_{\mu}\right)$ " could happen. The following three examples correspond to these three cases, respectively.

Example 1. Let the gradual set $\mu:\left[0, \frac{1}{2}\right) \rightarrow P(\mathbb{R}) \backslash\{\varnothing\}$ be defined as

$$
\mu(\alpha)=[\alpha-1,1-\alpha] \text { for } \alpha \in\left[0, \frac{1}{2}\right)
$$

Then, $\mu$ is strictly nested and:

$$
F_{\mu}(x)= \begin{cases}1+x, & x \in[-1,-1 / 2] \\ 1 / 2, & x \in[-1 / 2,1 / 2] \\ 1-x, & x \in[1 / 2,1] \\ 0, & \text { otherwise. }\end{cases}
$$

Thus, $[0,1 / 2]=\mathcal{R}\left(F_{\mu}\right) \supsetneqq I=[0,1 / 2)$.

Example 2. Let the gradual set $\mu:[0,1] \rightarrow P(\mathbb{R}) \backslash\{\varnothing\}$ be defined as

$$
[\mu]_{\alpha}= \begin{cases}{[-1 / 2,1-\alpha),} & \alpha \in[1 / 2,1], \\ {[\alpha-1,1 / 2),} & \alpha \in[0,1 / 2] .\end{cases}
$$

Then, $\mu$ is strictly nested and:

$$
F_{\mu}(x)= \begin{cases}1+x, & x \in(-1,-1 / 2) \\ 1, & x=[-1 / 2,0] \\ 1-x, & x \in(0,1 / 2) \\ 0, & \text { otherwise. }\end{cases}
$$

Thus, $[0,1 / 2) \cup(1 / 2,1]=\mathcal{R}\left(F_{\mu}\right) \varsubsetneqq I=[0,1]$.

Example 3. Let the gradual set $\mu:[0,1) \rightarrow P(\mathbb{R}) \backslash\{\varnothing\}$ be defined as

$$
[\mu]_{\alpha}= \begin{cases}{[-1 / 2,1-\alpha),} & \alpha \in[1 / 2,1), \\ {[\alpha-1,1 / 2),} & \alpha \in[0,1 / 2] .\end{cases}
$$

Then, $I=[0,1), \mu$ is strictly nested and:

$$
F_{\mu}(x)= \begin{cases}1+x, & x \in(-1,-1 / 2) \\ 1, & x=[-1 / 2,0] \\ 1-x, & x \in(0,1 / 2) \\ 0, & \text { otherwise. }\end{cases}
$$

Thus, $\mathcal{R}\left(F_{\mu}\right)=[0,1 / 2) \cup(1 / 2,1]$ and $I_{F_{\mu}}=[0,1]$. Therefore, $\mathcal{R}\left(F_{\mu}\right) \nsubseteq I$ and $I \nsubseteq \mathcal{R}\left(F_{\mu}\right)$.

We have the following conclusions on the relationship between $\mathcal{R}\left(F_{\mu}\right)$ and $I$, and the relationship between $I_{F_{\mu}}$ and $I$.

Proposition 2. Given a gradual set $\mu: I \rightarrow P(X) \backslash\{\varnothing\}$, then:

$$
\mathcal{R}\left(F_{\mu}\right) \backslash\{0\} \subseteq \mathbf{c l}\{I \backslash\{0\}\},
$$




$$
\widehat{I} \subseteq I_{F_{\mu}} \subseteq[0, \sup I],
$$

where $\widehat{I}=\{\alpha \in[0,1]: \alpha \leq \beta$ for some $\beta \in I\}$.

Proof. If $F_{\mu}(x)>0$, then there exists $\left\{\alpha_{n}\right\} \subseteq I \backslash\{0\}$, such that $x \in \mu\left(\alpha_{n}\right)$ and $\sup \alpha_{n}=$ $F_{\mu}(x)$. Thus, we know $\mathcal{R}\left(F_{\mu}\right) \backslash\{0\} \subseteq \mathbf{c l}\{I \backslash\{0\}\}$ and $I_{F_{\mu}} \subseteq[0$, sup $I]$.

If $x \in \mu(\alpha)$ for some $\alpha \in I$, then $F_{\mu}(x) \geq \alpha$. Therefore, we have $\widehat{I} \subseteq I_{F_{\mu}}$.

Remark 5. Note that $[0, \sup I] \backslash \widehat{I} \subseteq\{\sup I\}$. Thus, by Proposition $2, I_{F_{\mu}}$ has two possibilities: $\widehat{I}$ and $[0$, sup $I]$ (in some cases, these two are the same).

Clearly, if sup $I \in I$, then sup $I \in \mathcal{R}\left(F_{\mu}\right)$.

Even gradual set $\mu$ satisfies the conditions $\left(i^{\prime}\right)$ and $\left(i i^{\prime}\right)$ in Remark 4 , and each " $\subseteq$ " in Proposition 2 could be " $\subsetneq$ ".

Example 3 shows that $\mathcal{R}\left(F_{\mu}\right) \backslash\{0\} \varsubsetneqq \mathbf{c l}\{I \backslash\{0\}\}$ and $\widehat{I} \varsubsetneqq I_{F_{\mu}}$ could happen. The following Example 4 shows that $I_{F_{\mu}} \varsubsetneqq[0$, sup $I]$ could happen.

Example 4. Let the gradual set $\mu:[0,1) \rightarrow P(\mathbb{R}) \backslash\{\varnothing\}$ be defined as $\mu(\alpha)=[\alpha, 1)$. Then,

$$
F_{\mu}(x)= \begin{cases}x, & x \in[0,1), \\ 0, & \text { otherwise. }\end{cases}
$$

So $\mathcal{R}\left(F_{\mu}\right)=I_{F_{\mu}}=[0,1) \varsubsetneqq[0,1]=[0, \sup I]$.

Remark 6. Let $\mu: I \rightarrow P(X) \backslash\{\varnothing\}$ be a gradual set. Note that $I \subseteq \widehat{I}$. Thus, by Proposition 2, $I \subseteq I_{F_{\mu}}$.

From Remark 4, we can see that even conditions $\left(i^{\prime}\right)$ and $\left(i i^{\prime}\right)$ are satisfied, then $I \varsubsetneqq I_{F_{\mu}}$ could happen. Clearly, at this time, $\mathcal{R}\left(F_{\mu}\right) \nsubseteq I$.

In fact, if $\mu$ satisfies condition $\left(i^{\prime}\right)$, then $I \varsubsetneqq I_{F_{\mu}} \Leftrightarrow \mathcal{R}\left(F_{\mu}\right) \nsubseteq I \Leftrightarrow \sup I \notin I$ and $\sup I \in I_{F_{\mu}}$ $\Leftrightarrow \sup I \notin I$ and $\sup I \in \mathcal{R}\left(F_{\mu}\right)$.

\section{Interval Ranges of Compositions of Arithmetic}

It was claimed in [15] that $I_{A \circ B}$ is equal to $I^{\bigcap}$. In this section, we point out by examples that this conclusion should be modified. Furthermore, based on the results in Sections 3 and 4 , we discussed the relationship between $I_{\widetilde{f}\left(A_{1}, \ldots, A_{n}\right)}$ and $I^{\cap}$.

Note that either $I^{\cap} \subseteq I_{\widetilde{f}\left(A_{1}, \ldots, A_{n}\right)}$ or $I^{\cap} \supsetneqq I_{\widetilde{f}\left(A_{1}, \ldots, A_{n}\right)}$. We give the characterizations of all these two cases, respectively. Moreover, we give the relationship between $I_{\widetilde{f}}\left(A_{1}, \ldots, A_{n}\right)$ and $I^{\cap}$ in some particular cases. As a corollary of the above results, we illustrate the relationship between $I_{A \circ B}$ and $I^{\cap}$. by $I^{\cap}$.

The conclusions in this section show that $I_{\widetilde{f}\left(A_{1}, \ldots, A_{n}\right)}$ vary in the scope determined

Affirmation 2 ([15]). (see Section 4 of [15]) Suppose that $A, B \in F(\mathbb{R})$ and $\circ \in\{+,-, \times, /\}$. Then, $I_{A \circ B}=I^{\cap}$ (here $I^{\cap}=I_{A} \cap I_{B}$ ).

It can be checked that the Affirmation 2 is equivalent to the following Affirmation 3.

Affirmation 3. Supposing that $f$ is a composite of the finite arithmetic operations of real numbers and that $A_{i} \in F(\mathbb{R}), i=1, \ldots, n$. Then, $I_{\widetilde{f}\left(A_{1}, \ldots, A_{n}\right)}=I^{\cap}$ (here $I^{\bigcap}=\bigcap_{i=1}^{n} I_{A_{i}}$ ).

Affirmation 2 is not valid. The following example shows that, for each $\circ \in\{+,-, \times, /\}$, $I^{\cap} \varsubsetneqq I_{A \circ B}$ could happen. 
Example 5. Let $A$ be a fuzzy set in $\mathbb{R}$ defined as

$$
A(x)= \begin{cases}-0.5 x+1.5, & x \in(1,3) \\ 0, & \text { otherwise }\end{cases}
$$

and let $B$ be a fuzzy set in $\mathbb{R}$ defined as

$$
B(x)= \begin{cases}0.5 x+1.5, & x \in(-3,-1) \\ -0.5 x+1.5, & x \in(1,3) \\ 1.5-50 / x, & x \in(100 / 3,100) \\ 0, & \text { otherwise. }\end{cases}
$$

Then, $I_{A}=I_{B}=[0,1)$ and hence $I^{\cap}=[0,1)$.

Since:

$$
[A]_{\alpha}= \begin{cases}(1,3-2 \alpha], & \alpha \in(0,1) \\ \varnothing, & \alpha=1\end{cases}
$$

and:

$$
[B]_{\alpha}= \begin{cases}{[2 \alpha-3,-1) \cup(1,3-2 \alpha] \cup[100 /(3-2 \alpha), 100),} & \alpha \in(0,1), \\ \varnothing, & \alpha=1,\end{cases}
$$

we have for all $\alpha \in(0,1)$ :

$$
\begin{gathered}
0 \in[A]_{\alpha}+[B]_{\alpha}, \\
0 \in[A]_{\alpha}-[B]_{\alpha}, \\
100 \in[A]_{\alpha} \times[B]_{\alpha}, \\
1 \in[A]_{\alpha} /[B]_{\alpha} .
\end{gathered}
$$

So by Remark 3:

$$
\begin{gathered}
(A+B)(0)=1, \\
(A-B)(0)=1, \\
(A \times B)(100)=1, \\
(A / B)(1)=1,
\end{gathered}
$$

and thus:

$$
\begin{aligned}
I^{\cap} & =[0,1) \varsubsetneqq[0,1]=I_{A+B}, \\
I^{\cap} & =[0,1) \varsubsetneqq[0,1]=I_{A-B}, \\
I^{\cap} & =[0,1) \varsubsetneqq[0,1]=I_{A \times B}, \\
I^{\cap} & =[0,1) \varsubsetneqq[0,1]=I_{A / B} .
\end{aligned}
$$

Now we discuss the relationship between $I^{\cap}$ and $I_{\widetilde{f}\left(A_{1}, \ldots, A_{n}\right)}$. Their relationship can be divided into two cases: $I^{\cap} \subseteq I_{\widetilde{f}\left(A_{1}, \ldots, A_{n}\right)}$ or $I^{\cap} \supsetneqq I_{\widetilde{f}\left(A_{1}, \ldots, A_{n}\right)}$. The following Theorems 2 and 3 give characterizations of these two cases, respectively.

Theorem 2. Let $f$ be a composite of finite arithmetic operations of real numbers and for each $i=1, \ldots, n$, let $A_{i}$ be a fuzzy set in $F(\mathbb{R})$. Then, $I_{\widetilde{f}\left(A_{1}, \ldots, A_{n}\right)} \subseteq \operatorname{cl}\left\{I^{\cap}\right\}$ and the following statements are equivalent:

(i) $\quad I^{\cap} \subseteq I_{\widetilde{f}\left(A_{1}, \ldots, A_{n}\right)}$.

(ii) $I^{\cap} \subseteq I_{\widetilde{f}\left(A_{1}, \ldots, A_{n}\right)} \subseteq \mathbf{c l}\left\{I^{\cap}\right\}$.

(iii) $A_{i}=\bar{\varnothing}$ for some $i \in\{1, \ldots, n\}$ or $\widetilde{f}\left(A_{1}, \ldots, A_{n}\right) \neq \bar{\varnothing}$.

Proof. If $\widetilde{f}\left(A_{1}, \ldots, A_{n}\right)=\bar{\varnothing}$, then $I_{\widetilde{f}\left(A_{1}, \ldots, A_{n}\right)}=\{0\} \subseteq \operatorname{cl}\left\{I^{\cap}\right\}$. 
Let $\mu$ be the assignment function from $I^{\cap}$ to $P(\mathbb{R})$ defined by $\mu(\alpha)=f\left(\left[A_{1}\right]_{\alpha}, \ldots,\left[A_{n}\right]_{\alpha}\right)$. If $\widetilde{f}\left(A_{1}, \ldots, A_{n}\right) \neq \bar{\varnothing}$, then by Theorem $1, \widetilde{f}\left(A_{1}, \ldots, A_{n}\right)=F_{\mu}$. Thus, by Proposition 2, $I_{\widetilde{f}\left(A_{1}, \ldots, A_{n}\right)} \subseteq\left[0, \sup I^{\cap}\right]=\operatorname{cl}\left\{I^{\cap}\right\}$.

Therefore, $I_{\widetilde{f}\left(A_{1}, \ldots, A_{n}\right)} \subseteq \mathbf{c l}\left\{I^{\bigcap}\right\}$, and from this fact, we have (i) $\Leftrightarrow$ (ii).

If (iii) holds, then by Theorem $1, \widetilde{f}\left(A_{1}, \ldots, A_{n}\right)=F_{\mu}$. Note that $\widehat{I^{\cap}}=I^{\cap}$ and $\left[0, \sup I^{\cap}\right]=\operatorname{cl}\left\{I^{\cap}\right\}$. Therefore, by Proposition 2, (ii) holds. Thus, (iii) $\Rightarrow$ (ii).

Suppose $I^{\cap} \subseteq I_{\widetilde{f}\left(A_{1}, \ldots, A_{n}\right)}$. If $A_{i} \neq \bar{\varnothing}$ for all $i \in\{1, \ldots, n\}$, then $\{0\} \varsubsetneqq I^{\cap}$, and therefore $\{0\} \varsubsetneqq I_{\widetilde{f}\left(A_{1}, \ldots, A_{n}\right)}$. Thus, $\widetilde{f}\left(A_{1}, \ldots, A_{n}\right) \neq \bar{\varnothing}$. Therefore, we have (i) $\Rightarrow$ (iii).

Theorem 3. Let $f$ be a composite of finite arithmetic operations of real numbers and for each $i=1, \ldots, n$, let $A_{i}$ be a fuzzy set in $F(\mathbb{R})$. Then, the following statements are equivalent:

(i) $I^{\cap} \supsetneqq I_{\widetilde{f}\left(A_{1}, \ldots, A_{n}\right)}$.

(ii) $I^{\cap} \supsetneqq\{0\}$ and $I_{\widetilde{f}\left(A_{1}, \ldots, A_{n}\right)}=\{0\}$.

(iii) $A_{i} \neq \bar{\varnothing}$ for all $i \in\{1, \ldots, n\}$ and $\widetilde{f}\left(A_{1}, \ldots, A_{n}\right)=\bar{\varnothing}$.

Proof. The desired results follow immediately from Theorem 2.

The situation described in clause (iii) of Theorem 3 could happen. The following Example 6 gives an example of $A \neq \bar{\varnothing}, B \neq \bar{\varnothing}$ and $A / B=\bar{\varnothing}$.

Example 6. Let $A$ and $B$ be fuzzy sets in $F(\mathbb{R})$ defined as $A=\overline{\{1\}}$ and:

$$
B(x)= \begin{cases}1, & x=0 \\ 0.6, & x=1, \\ 0, & \text { otherwise. }\end{cases}
$$

Then, $I^{\cap}=[0,1]$. Note that there is no gradual number $a, b$ with $a \in A$ and $b \in B$ with $a / b$ being meaningful. Hence, the assignment function $\sigma$ corresponding to $A / B$ satisfies $\sigma(\alpha)=\varnothing$ for all $\alpha \in[0,1]$. Therefore, from (4) or (5), $A / B=\bar{\varnothing}$.

Clearly, at this time, $I^{\cap}=[0,1] \supsetneqq\{0\}=I_{A / B}$. lar cases.

Theorem 4 illustrates the relationship between $I^{\cap}$ and $I_{\widetilde{f}\left(A_{1}, \ldots, A_{n}\right)}$ in some particu-

Theorem 4. Let $f$ be a composite of finite arithmetic operations of real numbers and for each $i=1, \ldots, n$, let $A_{i}$ be a fuzzy set in $F(\mathbb{R})$ :

(i) If $A_{i}=\bar{\varnothing}$ for some $i \in\{1, \ldots, n\}$, then $\widetilde{f}\left(A_{1}, \ldots, A_{n}\right)=\bar{\varnothing}$. At this time, $I^{\cap}=$ $I_{\widetilde{f}\left(A_{1}, \ldots, A_{n}\right)}=\mathbf{c l}\left\{I^{\cap}\right\}=\{0\}$.

(ii) If $f$ is a composition of $\circ \in\{+,-, \times\}$, then $I^{\cap} \subseteq I_{\widetilde{f}\left(A_{1}, \ldots, A_{n}\right)} \subseteq \mathbf{c l}\left\{I^{\cap}\right\}$.

Proof. (i) obviously holds.

Suppose that $f$ is a composition of $\circ \in\{+,-, \times\}$. Let $\mu$ be the assignment function from $I^{\cap}$ to $P(\mathbb{R})$ defined by $\mu(\alpha)=f\left(\left[A_{1}\right]_{\alpha}, \ldots,\left[A_{n}\right]_{\alpha}\right)$. Then, by Theorem $1, \widetilde{f}\left(A_{1}, \ldots, A_{n}\right)=$ $F_{\mu}$. Note that $\widehat{I^{\cap}}=I^{\cap}$ and $\left[0, \sup I^{\bigcap}\right]=\operatorname{cl}\left\{I^{\cap}\right\}$. Thus, by Proposition 2, (ii) holds.

Remark 7. (ii) of Theorem 4 can also be proved as follows:

Suppose that $f$ is a composition of $\circ \in\{+,-, \times\}$ and that for each $i=1, \ldots, n, A_{i}$ is a fuzzy set in $F(\mathbb{R})$. Note that either $A_{i}=\bar{\varnothing}$ for some $i \in\{1, \ldots, n\}$ or $\widetilde{f}\left(A_{1}, \ldots, A_{n}\right) \neq \bar{\varnothing}$. Thus, by Theorem 2, the (ii) of Theorem 4 holds.

We have the following conclusions on $I_{A \circ B}$ and $I^{\cap}$.

Corollary 1. Let $A$ and $B$ be fuzzy sets in $F(\mathbb{R})$ : 
(i) If $\circ \in\{+,-, \times\}$, then $I^{\cap} \subseteq I_{A \circ B} \subseteq \operatorname{cl}\left\{I^{\cap}\right\}$.

(ii) $I^{\cap} \subseteq I_{A / B} \subseteq \mathbf{c l}\left\{I^{\cap}\right\}$ if and only if at least one of the following three items holds: $A=\bar{\varnothing}$, $B=\bar{\varnothing}$, and $A / B \neq \bar{\varnothing}$.

Proof. The desired results follow from Theorems 2 and 4 .

For each $\circ \in\{+,-, \times, /\}, I_{A \circ B}=I^{\cap}$ and $I_{A \circ B} \neq \mathbf{c l}\left\{I^{\cap}\right\}$ could happen. The following Example 7 is a such example.

Example 7. Consider $A$ in $F(\mathbb{R})$ defined by

$$
A(x)= \begin{cases}x, & x \in[0,1) \\ 0, & \text { otherwise. }\end{cases}
$$

Then, $I_{A}=[0,1)$.

It can be checked that:

$$
I_{A+\overline{\{1\}}}=I_{A-\overline{\{1\}}}=I_{A \times \overline{\{1\}}}=I_{A / \overline{\{1\}}}=I^{\cap}=[0,1) \varsubsetneqq[0,1]=\mathbf{c l}\left\{I^{\cap}\right\} .
$$

Remark 8. From Theorems 2 and 3, examples in this section and the fact $\mathbf{c l}\left\{I^{\cap}\right\} \backslash I^{\cap} \subseteq\{\sup I\}$, we have:

(i) $I_{\widetilde{f}\left(A_{1}, \ldots, A_{n}\right)}$ has three possibilities: $\{0\}, I^{\cap}$ and $\mathbf{c l}\left\{I^{\cap}\right\}$ (in some cases, all or part of these three are the same).

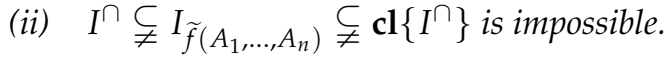

(iii) If $I^{\cap}=\mathbf{c l}\left\{I^{\cap}\right\}$ and $I_{\widetilde{f}\left(A_{1}, \ldots, A_{n}\right)} \neq\{0\}$, then $I^{\cap}=I_{\widetilde{f}\left(A_{1}, \ldots, A_{n}\right)}=\mathbf{c l}\left\{I^{\cap}\right\}$.

(ii) and (iii) can also be seen as corollaries of (i).

\section{Discussions on Ranges and Interval Ranges of Compositions of Arithmetic}

In [15], it is claimed that $I_{\widetilde{f}\left(A_{1}, \ldots, A_{n}\right)}, \mathcal{R}\left(\widetilde{f}\left(A_{1}, \ldots, A_{n}\right)\right)$ and $I^{\cap}$ are equal under certain conditions. In this section, we show by examples that this affirmation is not valid.

$\mathcal{R}\left(\widetilde{f}\left(A_{1}, \ldots, A_{n}\right)\right) \neq I^{\cap}$ can be divided into three cases: " $\mathcal{R}\left(\widetilde{f}\left(A_{1}, \ldots, A_{n}\right)\right) \supsetneqq I^{\cap ",}$ “R $\left(\widetilde{f}\left(A_{1}, \ldots, A_{n}\right)\right) \varsubsetneqq I^{\cap "}$ and " $\mathcal{R}\left(\widetilde{f}\left(A_{1}, \ldots, A_{n}\right)\right) \varsubsetneqq I^{\cap}$ and $I^{\cap} \nsupseteq \mathcal{R}\left(\widetilde{f}\left(A_{1}, \ldots, A_{n}\right)\right)$ ".

In this section, it is shown that even under stronger conditions than in [15], all the above three cases of $\mathcal{R}\left(\widetilde{f}\left(A_{1}, \ldots, A_{n}\right)\right) \neq I^{\cap}$ could happen.

$I_{\widetilde{f}\left(A_{1}, \ldots, A_{n}\right)}$ has three possibilities: $\{0\}, I^{\cap}$ and $\operatorname{cl}\left\{I^{\cap}\right\}$ (see Remark 8). $I_{\widetilde{f}\left(A_{1}, \ldots, A_{n}\right)} \neq I^{\cap}$ can be divided into two cases: " $I_{\widetilde{f}\left(A_{1}, \ldots, A_{n}\right)} \neq I^{\cap}$ and $I_{\widetilde{f}\left(A_{1}, \ldots, A_{n}\right)}=\{0\}$ " and " $I_{\widetilde{f}\left(A_{1}, \ldots, A_{n}\right)} \neq$ $I^{\cap}$ and $I_{\widetilde{f}\left(A_{1}, \ldots, A_{n}\right)}=\mathbf{c l}\left\{I^{\cap}\right\}^{\prime \prime}$.

In this section, it is shown that under the conditions in [15], both the above two cases of $I_{\widetilde{f}\left(A_{1}, \ldots, A_{n}\right)} \neq I^{\cap}$ could happen, and even under stronger conditions than in [15], "I $I_{\widetilde{f}\left(A_{1}, \ldots, A_{n}\right)} \neq I^{\cap}$ and $I_{\widetilde{f}\left(A_{1}, \ldots, A_{n}\right)}=\mathbf{c l}\left\{I^{\cap}\right\}$ " could happen.

The conclusions in this section show that even under stronger conditions than in [15], $I_{\widetilde{f}\left(A_{1}, \ldots, A_{n}\right)}, \mathcal{R}\left(\widetilde{f}\left(A_{1}, \ldots, A_{n}\right)\right)$ and $I^{\cap}$ may still be unequal.

A fuzzy set $A$ is said to be a canonical fuzzy set if $[A]_{\alpha} \neq[A]_{\beta}$ for all $\alpha, \beta \in[0,1]$ with $\alpha \neq \beta$.

Affirmation 4 ([15]). (See Proposition 4.5 of [15].) Let $f$ be a composite of finite arithmetic operations of real numbers and for each $i=1, \ldots, n$, let $A_{i}$ be a fuzzy set in $\mathbb{R}$. If some of $A_{1}, \ldots, A_{n}$ are canonical fuzzy sets in $\mathbb{R}$, then the assignment function $\mu: I^{\cap} \rightarrow P(\mathbb{R})$ defined by $\mu(\alpha)=f\left(\left[A_{1}\right]_{\alpha}, \ldots,\left[A_{n}\right]_{\alpha}\right)$ is strictly nested, and therefore:

$$
\mathcal{R}\left(\widetilde{f}\left(A_{1}, \ldots, A_{n}\right)\right)=I_{\widetilde{f}\left(A_{1}, \ldots, A_{n}\right)}=I^{\cap} .
$$


Affirmation 4 is not valid. This can be drawn from the facts listed below. Note that if $A$ is a canonical fuzzy set, then $I_{A}=[0,1]$ or $[0,1)$.

- $\quad$ There exist canonical fuzzy sets $A_{1}, \ldots, A_{n}$ such that $\tilde{f}\left(A_{1}, \ldots, A_{n}\right)=\bar{\varnothing}$. In this case, $I^{\cap}=[0,1]$ or $[0,1)$, and $\mathcal{R}\left(\widetilde{f}\left(A_{1}, \ldots, A_{n}\right)\right)=I_{\widetilde{f}\left(A_{1}, \ldots, A_{n}\right)}=\{0\}$. Therefore, $\mathcal{R}\left(\widetilde{f}\left(A_{1}, \ldots, A_{n}\right)\right) \neq I^{\cap}$ and $I_{\widetilde{f}\left(A_{1}, \ldots, A_{n}\right)} \neq I^{\cap}$.

- $\quad$ Let $A$ be a canonical fuzzy set. Then, $A \circ \overline{\mathbb{R}}=\overline{\mathbb{R}}$, where $\circ \in\{+,-, \times, /\}$. In this case, $I^{\cap}=I_{A} \cap I_{\mathbb{R}}=[0,1]$ or $[0,1), \mathcal{R}(A \circ \overline{\mathbb{R}})=\{1\}$ and $I_{A \circ \overline{\mathbb{R}}}=[0,1]$. Thus, $\mathcal{R}(A \circ \overline{\mathbb{R}}) \neq I^{\cap}$. Furthermore, $I_{A \circ \overline{\mathbb{R}}} \neq I^{\cap}$ when $I^{\cap}=[0,1)$.

Affirmation 4 is still not valid when the above cases are excluded.

Consider conditions:

(a) $\tilde{f}\left(A_{1}, \ldots, A_{n}\right) \neq \bar{\varnothing}$, and

(b) for each $i=1, \ldots, n, A_{i}$ is a canonical fuzzy set in $\mathbb{R}$.

Then, conditions (a) and (b) are stronger than that in Affirmation 4. If $A_{1}, \ldots, A_{n}$ and $f$ satisfy conditions (a) and (b), then the above cases are excluded.

We will show that even $A_{1}, \ldots, A_{n}$ and $f$ satisfy conditions (a) and (b), the conclusions in Affirmation 4 do not necessarily hold.

The following Example 8 shows that $\mu$ is not necessarily strictly nested even if $A_{1}, \ldots, A_{n}$ and $f$ satisfy conditions (a) and (b).

Example 8. Let $A$ be a fuzzy set defined as

$$
[A]_{\alpha}=[2,6-3 \alpha] \cup[6,7]
$$

for $\alpha \in[0,1]$, and let $B$ be a fuzzy set defined as

$$
[B]_{\alpha}=[1,3-\alpha] \cup[3,9]
$$

for $\alpha \in[0,1]$. Then, $A, B$ are canonical fuzzy sets in $\mathbb{R}$.

Note that for all $\alpha \in[0,1]$ :

$$
[A]_{\alpha} \times[B]_{\alpha}=[2,63] .
$$

Thus, the gradual set $\mu:[0,1] \rightarrow P(\mathbb{R}) \backslash\{\varnothing\}$ given by $\mu(\alpha)=[A]_{\alpha} \times[B]_{\alpha}$ is not strictly nested.

Remark 9. For A, B in Example 8, by Remark 3, we have:

$$
A \times B=F_{\mu}=\overline{[2,63]} .
$$

Thus, $\{1\}=\mathcal{R}(A \times B) \varsubsetneqq I_{A \times B}=I_{A} \cap I_{B}=[0,1]$. Therefore, (6) does not necessarily hold that $A_{1}, \ldots, A_{n}$ and $f$ satisfy conditions $(a)$ and $(b)$.

Remark 10. In fact, even $A_{1}, \ldots, A_{n}$ and $f$ satisfy:

(a) $\widetilde{f}\left(A_{1}, \ldots, A_{n}\right) \neq \bar{\varnothing}$,

(b) for each $i=1, \ldots, n, A_{i}$ is a canonical fuzzy set in $\mathbb{R}$, and

(c) $\mu$ is strictly nested,

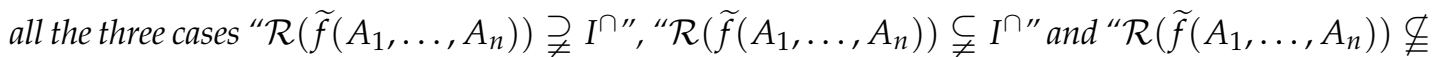
$I^{\cap}$ and $I^{\cap} \nsupseteq \mathcal{R}\left(\widetilde{f}\left(A_{1}, \ldots, A_{n}\right)\right)$ " of $\mathcal{R}\left(\widetilde{f}\left(A_{1}, \ldots, A_{n}\right)\right) \neq I^{\cap}$ could happen. The following three examples correspond to these three cases, respectively.

Example 9. Let $A$ be a fuzzy set in $\mathbb{R}$ defined as

$$
A(x)= \begin{cases}x, & x \in[0,1), \\ 0, & \text { otherwise. }\end{cases}
$$


Then, $I_{A}=[0,1)$ and:

$$
[A]_{\alpha}= \begin{cases}\varnothing, & \alpha=1 \\ {[\alpha, 1),} & 0<\alpha<1, \\ {[0,1],} & \alpha=0\end{cases}
$$

Hence, $A$ is a canonical fuzzy set, and the gradual set $\mu:[0,1) \rightarrow P(\mathbb{R}) \backslash\{\varnothing\}$ given by

$$
\mu(\alpha)=[A]_{\alpha}-[A]_{\alpha}= \begin{cases}(\alpha-1,1-\alpha), & 0<\alpha<1, \\ {[-1,1],} & \alpha=0,\end{cases}
$$

is strictly nested.

So, by Remark 3,

$$
(A-A)(x)=F_{\mu}(x)= \begin{cases}1+x, & x \in[-1,0] \\ 1-x, & x \in[0,1] \\ 0, & \text { otherwise }\end{cases}
$$

and thus

$$
[0,1]=\mathcal{R}(A-A)=I_{A-A} \supsetneqq I^{\bigcap}=[0,1) .
$$

Example 10. Let A be the fuzzy set derived from the gradual set given in Example 3; that is:

$$
[A]_{\alpha}= \begin{cases}{[-1 / 2,1-\alpha],} & \alpha \in(1 / 2,1] \\ {[\alpha-1,1 / 2),} & \alpha \in[0,1 / 2] .\end{cases}
$$

Hence, $A$ is a canonical fuzzy set and

$$
[A]_{\alpha}+[A]_{\alpha}= \begin{cases}{[-1,2-2 \alpha],} & \alpha \in(1 / 2,1] \\ {[2 \alpha-2,1),} & \alpha \in[0,1 / 2] .\end{cases}
$$

Therefore, we have the gradual set $\mu:[0,1] \rightarrow P(\mathbb{R}) \backslash\{\varnothing\}$ given by $\mu(\alpha)=[A]_{\alpha}+[A]_{\alpha}$ is strictly nested and by Remark 3:

$$
(A+A)(x)=F_{\mu}(x)= \begin{cases}1+x / 2, & x \in(-2,-1) \\ 1, & x=[-1,0] \\ 1-x / 2, & x \in(0,1) \\ 0, & \text { otherwise. }\end{cases}
$$

Thus, $[0,1 / 2) \cup(1 / 2,1]=\mathcal{R}(A+A) \varsubsetneqq I^{\bigcap}=I_{A+A}=[0,1]$.

Example 11. Let $A$ be the fuzzy set in $\mathbb{R}$ defined as

$$
A(x)= \begin{cases}x+1, & -1<x<-0.5 \\ 0.6, & x=-0.5 \\ -x+0.5, & -0.5<x<0 \\ 0, & \text { otherwise }\end{cases}
$$

and let $B$ be the fuzzy set in $\mathbb{R}$ defined as

$$
B(x)= \begin{cases}-x+0.5, & -0.5<x<0, \\ 0.6, & x=0 \\ -0.5 x+0.5, & 0<x<1 \\ 0, & \text { otherwise. }\end{cases}
$$


Then:

$$
[A]_{\alpha}= \begin{cases}{[-1,0],} & \alpha=0 \\ {[\alpha-1,0),} & 0<\alpha \leq 0.5 \\ {[-0.5,-\alpha+0.5],} & 0.5<\alpha \leq 0.6 \\ (-0.5,-\alpha+0.5], & 0.6<\alpha<1 \\ \varnothing, & \alpha=1\end{cases}
$$

and:

$$
[B]_{\alpha}= \begin{cases}{[-0.5,1],} & \alpha=0, \\ (-0.5,1-2 \alpha], & 0<\alpha \leq 0.5 \\ (-0.5,-\alpha+0.5] \cup\{0\}, & 0.5<\alpha \leq 0.6, \\ (-0.5,-\alpha+0.5], & 0.6<\alpha<1 \\ \varnothing, & \alpha=1 .\end{cases}
$$

Hence, $A$ and $B$ are canonical fuzzy sets, and:

$$
[A]_{\alpha}-[B]_{\alpha}= \begin{cases}{[-2,0.5],} & \alpha=0, \\ {[-2+3 \alpha, 0.5),} & 0<\alpha \leq 0.5 \\ {[-0.5,-\alpha+1),} & 0.5<\alpha \leq 0.6 \\ (-1+\alpha,-\alpha+1), & 0.6<\alpha<1 \\ \varnothing, & \alpha=1\end{cases}
$$

Therefore, we have the gradual set: $\mu:[0,1) \rightarrow P(\mathbb{R}) \backslash\{\varnothing\}$ given by $\mu(\alpha)=[A]_{\alpha}-[B]_{\alpha}$ which is strictly nested and:

$$
(A-B)(x)=F_{\mu}(x)= \begin{cases}(x+2) / 3, & -2<x<-0.5 \\ 0.6, & -0.5 \leq x \leq-0.4 \\ x+1, & -0.4<x \leq 0 \\ 1-x, & 0<x<0.5 \\ 0, & \text { otherwise. }\end{cases}
$$

Thus, $\mathcal{R}(A-B)=[0,0.5) \cup(0.5,1]$. Note that $I^{\cap}=[0,1)$. Therefore, we have $\mathcal{R}(A-$ B) $\nsubseteq I^{\cap}$ and $I^{\cap} \nsupseteq \mathcal{R}(A-B)$.

Remark 11. From the facts listed at the beginning of this section, we know that under the assumption of Affirmation 4, both the two cases " $I_{\widetilde{f}\left(A_{1}, \ldots, A_{n}\right)} \neq I^{\cap}$ and $I_{\widetilde{f}\left(A_{1}, \ldots, A_{n}\right)}=\{0\}$ " and "I $I_{\widetilde{f}\left(A_{1}, \ldots, A_{n}\right)} \neq I^{\cap}$ and $I_{\widetilde{f}\left(A_{1}, \ldots, A_{n}\right)}=\mathbf{c l}\left\{I^{\cap}\right\}$ " of $I_{\widetilde{f}\left(A_{1}, \ldots, A_{n}\right)} \neq I^{\cap}$ could happen.

We mention that, even $A_{1}, \ldots, A_{n}$ and $f$ satisfy:

(a) $\widetilde{f}\left(A_{1}, \ldots, A_{n}\right) \neq \bar{\varnothing}$,

(b) for each $i=1, \ldots, n, A_{i}$ is a canonical fuzzy set in $\mathbb{R}$, and

(c) $\mu$ is strictly nested,

$I_{\widetilde{f}\left(A_{1}, \ldots, A_{n}\right)} \neq I^{\cap}$ could happen, and clearly at this time, $I_{\widetilde{f}\left(A_{1}, \ldots, A_{n}\right)}=\mathbf{c l}\left\{I^{\cap}\right\}$, because condition (a) is equivalent to $I_{\widetilde{f}\left(A_{1}, \ldots, A_{n}\right)} \neq\{0\}$. See Example 9 for such an example.

In fact, under conditions (a), (b) and (c), $I_{\widetilde{f}\left(A_{1}, \ldots, A_{n}\right)}$ has two possibilities: $I^{\cap}$ and $\mathbf{c l}\left\{I^{\cap}\right\}$. and (c).

It can be seen that $I_{\widetilde{f}\left(A_{1}, \ldots, A_{n}\right)}=I^{\bigcap}=\operatorname{cl}\left\{I^{\bigcap}\right\}$ could happen under conditions (a), (b)

The following Example 12 shows that $I_{\widetilde{f}\left(A_{1}, \ldots, A_{n}\right)} \neq \mathbf{c l}\left\{I^{\cap}\right\}$ could happen while conditions (a), (b) and (c) are satisfied. Clearly, at this time, $I_{\widetilde{f}\left(A_{1}, \ldots, A_{n}\right)}=I^{\cap}$.

Example 12. Consider A given in Example 9. Then, $A$ is a canonical fuzzy set, and the gradual set $\mu:[0,1) \rightarrow P(\mathbb{R}) \backslash\{\varnothing\}$ given by

$$
\mu(\alpha)=[A]_{\alpha}+[A]_{\alpha}= \begin{cases}{[2 \alpha, 2),} & 0<\alpha<1 \\ {[0,2],} & \alpha=0\end{cases}
$$


is strictly nested.

Therefore, by Remark 3:

$$
(A+A)(x)=F_{\mu}(x)= \begin{cases}x / 2, & x \in[0,2) \\ 0, & \text { otherwise }\end{cases}
$$

and thus:

$$
[0,1)=I^{\cap}=I_{A+A}=\mathcal{R}(A+A) \neq \mathbf{c l}\left\{I^{\cap}\right\}=[0,1] .
$$

Remark 12. When $\mathcal{R}\left(\widetilde{f}\left(A_{1}, \ldots, A_{n}\right)\right)=I_{\widetilde{f}\left(A_{1}, \ldots, A_{n}\right)}=I^{\cap}, I^{\cap} \neq \operatorname{cl}\left\{I^{\cap}\right\}$ could happen, and clearly in this case, $I^{\cap} \neq\{0\}$. See Example 12 for a such example.

We can see that when $\mathcal{R}\left(\widetilde{f}\left(A_{1}, \ldots, A_{n}\right)\right)=I_{\widetilde{f}\left(A_{1}, \ldots, A_{n}\right)}=I^{\cap}, I^{\cap}=\operatorname{cl}\left\{I^{\cap}\right\}$ also could happen, and in this case, both $I^{\cap}=\{0\}$ and $I^{\cap} \neq\{0\}$ are possible.

Remark 13. The content of this paper is interrelated. For instance, the conclusion in Remark 10 implies the conclusion in Remark 4. Examples 9, 10 and 11 also show the conclusion in Remark 4. Example 5 can be used to illustrate some of the relationship between $I_{\widetilde{f}}\left(A_{1}, \ldots, A_{n}\right)$ and $I^{\cap}$ given in this paper, and some of the relationship between $\mathcal{R}\left(\widetilde{f}\left(A_{1}, \ldots, A_{n}\right)\right)$ and $I^{\cap}$ given in this paper.

\section{Conclusions}

In this paper, we give general conclusions on the problems related to the interval ranges of the resulted fuzzy sets of compositions of finite arithmetic.

In [15], it is claimed that under certain conditions, the domain $I$ of a gradual set $\mu$ is equal to the range $\mathcal{R}\left(F_{\mu}\right)$ of its induced fuzzy set $F_{\mu}$.

We show by examples that this is not valid. In fact, even under stronger conditions than in [15], there are still various possibilities in the relationship between $I$ and $\mathcal{R}\left(F_{\mu}\right)$. Moreover, we give the relationship between $I$ and $\mathcal{R}\left(F_{\mu}\right)$, and the relationship between $I$ and $I_{F_{\mu}}$.

In [15], it is claimed that $I^{\cap}=I_{A \circ B}$ when $\circ$ is an arithmetic operation in $\{+,-, \times, /\}$.

We show by examples that this is not valid. Furthermore, we give the relationship between $I^{\cap}$ and $I_{\widetilde{f}\left(A_{1}, \ldots, A_{n}\right)}$. As a corollary, we give the relationship between $I^{\cap}$ and $I_{A \circ B}$ for $\circ \in\{+,-, \times, /\}$. We point out that $I_{\widetilde{f}\left(A_{1}, \ldots, A_{n}\right)}$ has three possibilities: $\{0\}, I^{\cap}$ and $\mathbf{c l}\left\{I^{\cap}\right\}$.

In [15], it is claimed that under certain conditions, $\mathcal{R}\left(\widetilde{f}\left(A_{1}, \ldots, A_{n}\right)\right)=I_{\widetilde{f}\left(A_{1}, \ldots, A_{n}\right)}=I^{\cap}$.

We show by examples that this is not valid. In fact, even under stronger conditions than in [15], there are still various possibilities in the relationship between $\mathcal{R}\left(\widetilde{f}\left(A_{1}, \ldots, A_{n}\right)\right)$ and $I^{\cap}$, and there are still various possibilities in the relationship between $I_{\widetilde{f}\left(A_{1}, \ldots, A_{n}\right)}$ and $I^{\cap}$.

The conclusions of this paper show that $I_{\widetilde{f}\left(A_{1}, \ldots, A_{n}\right)}$ and $\mathcal{R}\left(\widetilde{f}\left(A_{1}, \ldots, A_{n}\right)\right)$ vary in the scopes determined by $I^{\cap}$, respectively. On the other hand, even under stronger conditions than in [15], these and $I^{\cap}$ are not necessarily equal, and there exist various possibilities in their relationship with $I^{\cap}$.

The results in this paper can be used in the theoretical research and practical applications of gradual numbers, gradual sets and arithmetic using the gradual numbers and gradual sets introduced by $\mathrm{Wu}$ [15]. We will discuss the properties of this kind of arithmetic in the future.

Author Contributions: Formal analysis, Q.M.; methodology, H.H.; writing-original draft preparation, Q.M. and H.H.; writing-review and editing, H.H. All authors have read and agreed to the published version of the manuscript.

Funding: This research was funded by the Natural Science Foundation of Fujian Province of China (grant number 2020J01706).

Institutional Review Board Statement: Not applicable.

Informed Consent Statement: Not applicable. 
Data Availability Statement: Not applicable.

Acknowledgments: The authors would like to thank the anonymous reviewers for their valuable comments and suggestions which greatly improved the presentation of this paper.

Conflicts of Interest: The authors declare no conflict of interest.

\section{References}

1. Zadeh, L.A. Fuzzy Sets. Inform. Control 1965, 8, 338-353. [CrossRef]

2. Dubois, D.; Prade, H. Fundamentals of Fuzzy Sets, The Handbooks of Fuzzy Sets Series; Kluwer Academic Publishers: London, UK, 2000; Volume 7.

3. Popa, L.; Sida, L. Fuzzy Inner Product Space: Literature Review and a New Approach. Mathematics 2021, 9, 765. [CrossRef]

4. Xing, Y.; Qiu, D. Solving Triangular Intuitionistic Fuzzy Matrix Game by Applying the Accuracy Function Method. Symmetry 2019, 11, 1258. [CrossRef]

5. Fortin, J.; Dubois, D.; Fargier, H. Gradual numbers and their application to fuzzy interval analysis. IEEE Trans. Fuzzy Syst. 2008, 16, 388-401. [CrossRef]

6. Dubois, D.; Prade, H. Gradual elements in a fuzzy set. Soft Comput. 2008, 12, 165-175. [CrossRef]

7. Dubois, D.; Prade, H. Gradualness, uncertainty and bipolarity: Making sense of fuzzy sets. Fuzzy Sets Syst. 2012, 192, 3-24 [CrossRef]

8. Boukezzoula, R.; Galichet, S.; Foulloy, L.; Elmasry, M. Extended gradual interval arithmetic and its application to gradual weighted averages. Fuzzy Sets Syst. 2014, 257, 67-84. [CrossRef]

9. Boukezzoula, R.; Coquin, D. Interval-valued fuzzy regression: Philosophical and methodological issues. Appl. Soft Comput. 2021, 103, 107145. [CrossRef]

10. Pourabdollaha, A.; Mendel, J.M.; John, R.I. Alpha-cut representation used for defuzzification in rule-based systems. Fuzzy Sets Syst. 2020, 399, 110-132. [CrossRef]

11. Klir, G.J. Fuzzy arithmetic with requisite constraints. Fuzzy Sets Syst. 1997, 91, 165-175. [CrossRef]

12. Stefanini, L. A generalization of Hukuhara difference and division for interval and fuzzy arithmetic. Fuzzy Sets Syst. 2010, 161, 1564-1584. [CrossRef]

13. Chalco-Cano, Y.; Lodwick, W.A.; Bede, B. Single level constraint interval arithmetic. Fuzzy Sets Syst. 2014, 257, 146-168. [CrossRef]

14. Ngan, S.-C. A concrete reformulation of fuzzy arithmetic. Expert Syst. Appl. 2021, 167, 113818. [CrossRef]

15. Wu, H.-C. Arithmetic operations of non-normal fuzzy sets using gradual numbers. Fuzzy Sets Syst. 2020, 399, 1-19. [CrossRef]

16. Nguyen, H.T. A note on the extension principle for fuzzy sets. J. Math. Anal. Appl. 1978, 64, 369-380. [CrossRef]

17. Zadeh, L.A. The concept of linguistic variable and its application to approximate reasoning. Inform. Sci. 1975, 8, 199-249. [CrossRef]

18. Wu, C.; Ma, M. The Basic of Fuzzy Analysis; National Defence Industry Press: Beijing, China, 1991. (In Chinese)

19. Gong, Z.; Hao, Y. Fuzzy Laplace transform based on the Henstock integral and its applications in discontinuous fuzzy systems. Fuzzy Sets Syst. 2019, 358, 1-28. [CrossRef]

20. Wang, G.; Shi, P.; Wang, B.; Zhang, J. Fuzzy $n$-ellipsoid numbers and representations of uncertain multichannel digital information. IEEE Trans. Fuzzy Syst. 2014, 22, 1113-1126. [CrossRef]

21. Huang, H. Characterizations of endograph metric and $\Gamma$-convergence on fuzzy sets. Fuzzy Sets Syst. 2018, 350, 55-84. [CrossRef] 